\title{
SCIENTIFIC REPORTS

\section{OPEN Identification and characterization of the MADS-box genes highly expressed in the laticifer cells of Hevea brasiliensis}

Received: 20 August 2018

Accepted: 16 August 2019

Published online: 03 September 2019

\author{
Ying Wang ${ }^{1,2}$, Di-Feng Zhan ${ }^{1,3}$, Hui-Liang $\mathrm{Li}^{1,2}$, Dong Guo ${ }^{1,2}$, Jia-Hong Zhu ${ }^{1,2}$ \& \\ Shi-Oing Peng ${ }^{1,2}$
}

MADS-box transcription factors possess many functions in plant reproduction and development. However, few MADS-box genes related to secondary metabolites regulation have been identified. In Hevea brasiliensis, natural rubber is a representative cis-polyisoprenoids in secondary metabolism which occurs in the rubber laticifer cells, the molecular regulation basis of natural rubber biosynthesis is not clear. Here, a total of 24 MADS-box genes including 4 type I MADS-box genes and 20 type II MADS-box genes were identified in the transcriptome of rubber tree latex. The phylogenetic analysis was performed to clarify the evolutionary relationships of all the 24 rubber tree MADS-box proteins with MADS-box transcription factors from Arabidopsis thaliana and Oryza sativa. Four type I MADSbox genes were subdivided into $\mathrm{M \alpha}$ ( 3 genes) and $\mathrm{M} \beta$ ( 1 gene). Twenty type II MADS-box genes were subclassified into MIKC* (8 genes) and MIKC' (12 genes). Eight MADS-box genes (HblMADS3, 5, 6, 7, 9, $13,23,24)$ were predominant expression in laticifers. ABA up-regulated the expression of HbIMADS9, and the expression of HbIMADS3, HbIMADS5, HbIMADS24 were up-regulated by MeJA. The function of HbIMADS24 was elucidated. HbIMADS24 bound HbFPS1 promoter in yeast and HbIMADS24 activated HbFPS1 promoter in tobacco plants. Moreover, we proposed that HbIMADS24 is a transcription activator of HbFPS1 which taking part in natural rubber biosynthesis.

MADS-box transcription factors play an indispensable role in plant growth and development ${ }^{1-4}$. In plants, MADS-box genes possess many functions in determination of floral organ identity, floral transition, flowering time determination ${ }^{3,5-10}$, embryo development and seed pigmentation ${ }^{11}$, fruit ripening regulation ${ }^{12}$. The MADS-box gene family is classified into two major categories: type I and type $\mathrm{II}^{13,14}$. The type I MADS-box genes are classified into three subgroups including $\mathrm{M} \alpha, \mathrm{M} \beta$ and $\mathrm{M} \gamma$, whereas the type II MADS-box genes are subdivided into $\mathrm{MIKC}^{\mathrm{C}}$ and MIKC* types according to different exon and intron structures ${ }^{4,15,16}$. The $\mathrm{MIKC}^{\mathrm{C}}$ genes are subdivided into 12 clades according to the phylogeny in angiosperms ${ }^{17}$. MADS-box proteins contain a highly conserved MADS-boxdomain which is composed of about 60-amino-acid sequences that bind to CArG box (CC $\left.[\mathrm{A} / \mathrm{T}]_{6} \mathrm{GG}\right)^{18-21}$. In addition, the type II lineage includes three other domains: the Keratin-like (K) domain, the Intervening (I) domain and the C-terminal (C) region ${ }^{22-24}$. In dicot plants such as Arabidopsis, the floral homeotic genes were divided into ABCDE-classes based on function ${ }^{25-27}$. In monocot crops such as rice, two D class genes,

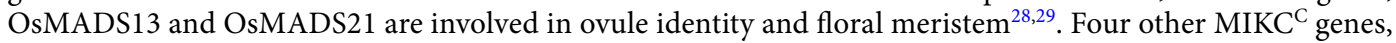
Suppressor of Overexpression of Constans1 (SOC1) $)^{30,31}$, Flowering Locus c (FLC) ${ }^{32,33}$, AGAMOUSLIKE GENE 24 (AGL24) $^{34,35}$ and Short Vegetative Phase (SVP) ${ }^{36}$ played a key role in flower initiation and flowering time. SHATTERPROOF $1-2$ and FUL related to fruit ripening ${ }^{12,26}$, TRANSPARENT TESTA16 act in endothelium development and seed pigmentation ${ }^{11}$.

Natural rubber (NR) is a cis-1, 4-polyisoprene biopolymer that is obtained commercially from the latex of rubber tree (Hevea brasiliensis Muell. Arg) ${ }^{37}$. NR biosynthesis is a branch of the isoprenoid pathway which

\footnotetext{
${ }^{1}$ Key Laboratory of Biology and Genetic Resources of Tropical Crops, Ministry of Agriculture, Institute of Tropical Bioscience and Biotechnology, Chinese Academy of Tropical Agricultural Sciences, Haikou, 571101, China. ${ }^{2}$ Hainan Academy of Tropical Agricultural Resource, CATAS, Haikou, 571101, China. ${ }^{3}$ Institute of Tropical Crops, Hainan University, Haikou, 570228, China. Correspondence and requests for materials should be addressed to S.-O.P. (email: shqpeng@163.com)
} 
occurs on the surface of the rubber particle in the rubber laticifer cells ${ }^{38,39}$. NR is biosynthesized by sequential condensations of isopentenyl diphosphates which are synthesized via the mevalonate pathway ${ }^{40-42}$. In the rubber tree, farnesyl diphosphate synthase (FPS) is an important enzyme in isoprenoids secondary metabolism. HbFPS1 expressed obviously in the laticifers which is possible to involve in NR biosynthesis. However, HbFPS2 and HbFPS 3 have no cell-type specific expression, and they are likely to act as housekeeping nature to involve in isoprenoid biosynthesis ${ }^{43}$.

In H. brasiliensis, the general NR biosynthesis metabolic pathway is now clear, but the molecular regulation of some NR biosynthesis-related genes is limited ${ }^{41,44}$. To date, few MADS-box genes related to NR biosynthesis have been identified. For instance, three MADS-box genes of $H$. brasiliensis were identified. They were differentially expressed in the laticifer cells ${ }^{45}$. HbMADS4 was identified to down-regulate the expression HbSRPP involved in NR biosynthesis ${ }^{46}$. In the present study, we identified and systematically analyzed the 24 MADS-box family genes (named HblMADS1 to HblMADS24) from the rubber tree latex transcriptome. Eight MADS-box genes were identified as predominantly expressed in laticifers. Furthermore, HblMADS24 positively regulated the HbFPS1 expression.

\section{Results}

Identification and phylogenetic analysis of the MADS-box genes from $\mathrm{H}$. brasiliensis. Our laboratory had obtained the $H$. brasiliensis latex transcriptome database by the Illumina HiSeq 2000 method $^{47}$. A total of 36 MADS-box unigenes were obtained by scanning the $H$. brasiliensis latex transcriptome database. These MADS-box unigenes were used as queries in BLAST against the local $\mathrm{H}$. brasiliensis genome database. A total of 24 MADS-box genes were confirmed from $H$. brasiliensis genome. These MADS-box genes were designated as HblMADS1 to HblMADS24, respectively (Additional file Table S1). The number of nucleotides of all the 24 MADS-box genes coding domain sequence varied from $522 \mathrm{bp}$ to $1101 \mathrm{bp}$, the number of amino acids encoded the 24 MADS-box proteins varied from 173 aa to 366 aa, and the predicted relative molecular mass ranged from 20.4 to $41.18 \mathrm{kDa}$, with protein isoelectric point (pI) in the range of 5.04 to 10.14 (Additional file Table S1).

To determine the evolutionary relationships between these MADS-box genes in rubber tree latex and other species, the phylogenetic tree was constructed among MADS-box genes from $H$. brasiliensis and known MADS-box proteins from Arabidopsis thaliana and Oryza sativa using the neighbor-joining method (Fig. 1). According to the phylogenetic analysis, 24 MADS-box genes from $H$. brasiliensis were classified into two groups, including type I MADS-box genes (4 genes) and type II MADS-box genes (20 genes). Four type I MADS-box genes were subdivided into $\mathrm{M} \alpha$ (3 genes) and $\mathrm{M} \beta$ (1 gene). Twenty type II MADS-box genes were subdivided into MIKC* (8 genes) and MIKC ${ }^{c}$ (12 genes). The 12 MIKC $^{c}$ genes were further classified into 5 subfamilies: SOC1 (4 genes), AGL17 (3 genes), SVP (2 genes), AP1 (2 genes) and FLC (1 gene).

Gene structure analysis and identification of conserved motifs of $H$. brasiliensis MADS-box genes. The exon/intron patterns of MADS-box genes from $H$. brasiliensis were analyzed by the online software GSDS. The genes of the same subfamily had significant similar exon-intron structures, differing only in the number of nucleotides of exon/intron (Fig. 2). In general, the type II MADS-box genes had more exons compared with the type I MADS-box genes, which suggested that the type II MADS-box genes have more complex gene structure. The number of exon of the twenty type II MADS-box genes ranged from 7 to 11, while four type I MADS-box genes contained only one exon. The MIKC* genes displayed less number of nucleotides and more exons than the MIKC ${ }^{\mathrm{C}}$ genes.

The conserved motifs of $24 \mathrm{HblMADS}$ proteins were analyzed to illuminate the features of MADS-box protein sequences by MEME motif search tool, resulting in the identification of 17 conserved motifs (Fig. 3 ). In the same subfamily, most of the closely related proteins distributed similar motif type. All the 24 HblMADS proteins contained motif 1 which is the most typical MADS-box domain in plant MADS-box proteins. Motif 3 represented the most conserved $\mathrm{K}$ domain, which was verified in all the type II HblMADS proteins. The K-domain was also only observed in other plants type II MADS-box proteins ${ }^{48}$.

Expression patterns of MADS-box genes in different tissues of $\boldsymbol{H}$. brasiliensis. The expression of all the 24 MADS-box genes was detected in five different tissues (roots, barks, leaves, flowers, latex) by quantitative real-time PCR (qRT-PCR). A heat map showed that different MADS-box genes had diverse expression patterns in different tissues (Fig. 4). Eight MADS-box genes (HblMADS3, 5, 6, 7, 9, 13, 23, 24) had higher expression levels in latex but relatively low expressed in other tissues. Eight MADS-box genes (HblMADS8, 12, 16, 17, $18,19,20,22)$ maintained significantly high expression level in the flowers, whereas eight MADS-box genes (HblMADS1, 2, 4, 10, 11, 14, 15, 21) in the leaves. By contrast, all MADS-box genes had no expression in the roots and lowly expressed in barks.

Expression analysis of MADS-box genes in the latex in response to hormone treatments. Eight MADS-box genes (HblMADS3, 5, 6, 7, 9, 13, 23, 24) maintained higher expression levels in latex. However, HblMADS6, HblMADS7, HblMADS13, HblMADS23 have been identified ${ }^{45,46}$. HblMADS3, HblMADS5, HblMADS9, HblMADS24 were analyzed in response to exogenous plant hormones. The expression patterns of these four genes were different under abscisic acid (ABA), ethrel (ET), methyl jasmonate (MeJA), and salicylic acid (SA) treatments (Fig. 5). The results indicated that MeJA treatment up-regulated the expression of HblMADS3, HblMADS5 and HblMADS24 at either $9 \mathrm{~h}$ or $12 \mathrm{~h}$ time points, while had no influence on that of HblMADS9. ABA treatment evidently up-regulated the expression of HblMADS9 at $12 \mathrm{~h}$, whereas had not significantly affected that of HblMADS3, HblMADS5 and HblMADS24. Under the SA treatment, the expression of HblMADS9 and HblMADS24 were slightly up-regulated at $9 \mathrm{~h}$ or $6 \mathrm{~h}$ time point, while had no significantly effect on that of HblMADS3 and HblMADS5. ET stress had no obvious influence on the expression of four HblMADS genes. 


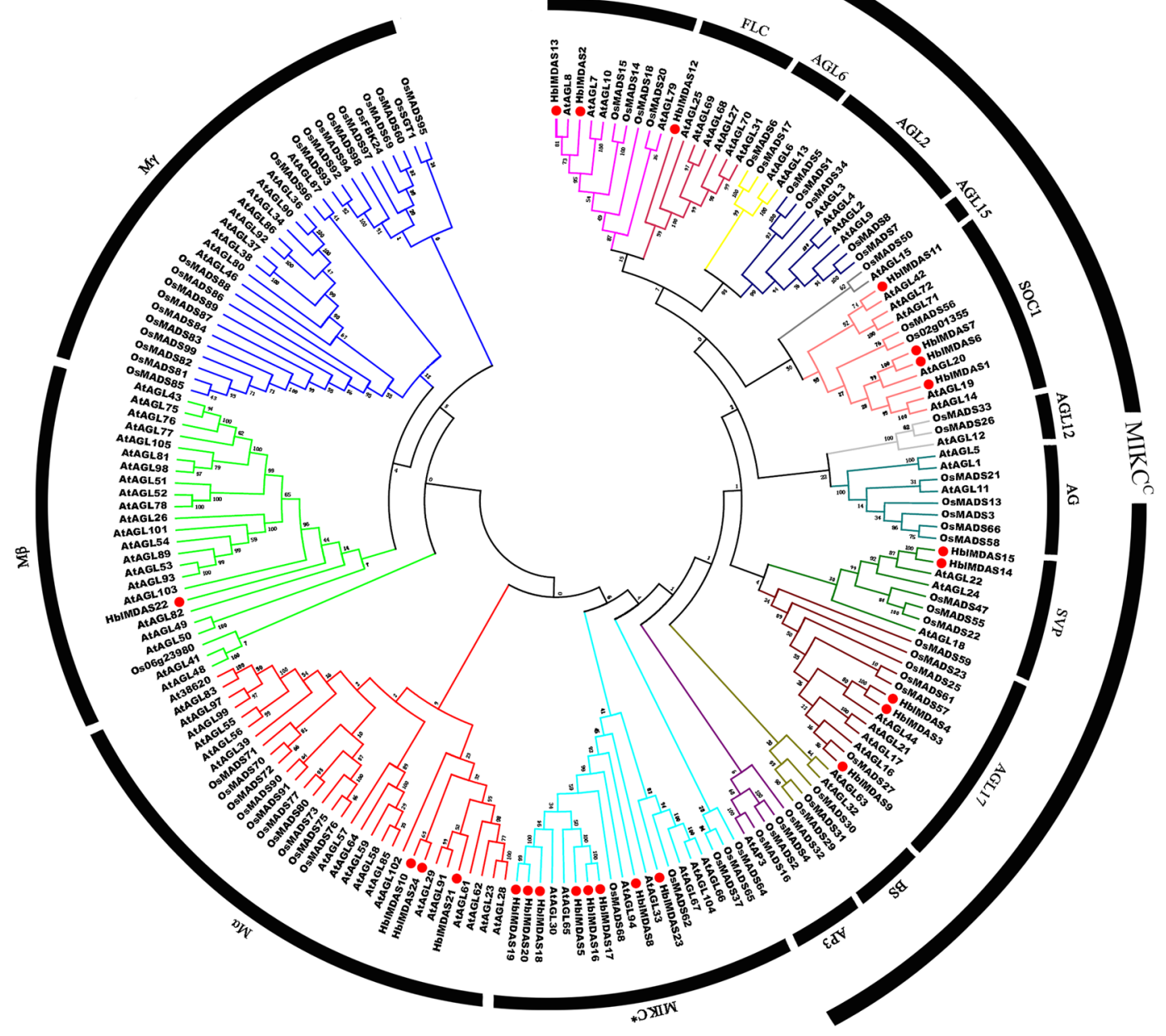

Figure 1. Phylogenetic analysis of the MADS proteins from H. brasiliensis with Arabidopsis and Oryza sativa MADS proteins. The phytozome database (https://phytozome.jgi.doe.gov/pz/portal.html) was used to download the Arabidopsis and O. sativa MADS protein sequences. The phylogenetic tree was constructed among all MADS proteins from $H$. brasiliensis, Arabidopsis and O. sativa using the neighbor-joining method, and bootstrap analysis were set to 1,000 replications using MEGA6.0. The HblMADS proteins are indicated by red dots. The subgroups are marked by black lines.

Subcellular localization of HbIMADS24. To elucidate the function of HblMADS24, we performed the subcellular localization analysis on HblMADS24. We found that the green fluorescent protein (GFP) tagged HblMADS24 was indeed present in the nucleus of onion epidermal cells, while GFP alone exhibited a cytoplasmic distribution (Fig. 6).

Activation of the HbFPS1 promoter by HbIMADS24 in yeast. HbFPS1 expressed obviously in the laticifers which is possible to involve in natural rubber biosynthesis ${ }^{43}$. The yeast one-hybrid analysis was performed to investigate whether HblMADS24 binds the HbFPS1 promoter. The yeast clones harboring pGAD-HblMADS24 and pHis-pHbFPS1 could grow on triple dropout medium lacking histidine, tryptophan, and leucine (SD/-Trp/-His/-Leu) containing $70 \mathrm{mM} 3$-amino-1, 24-triazole (3-AT), suggesting HblMADS24 bound the HbFPS1 promoter in yeast (Fig. 7).

Activation of the HbFPS1 promoter by HbIMADS24 in plants. Since HblMADS24 was able to interact with the HbFPS1 promoter in yeast, the Dual-LUC method was used to investigate whether HblMADS24 can regulate the HbFPS1 promoter in plants. The reporter strain pGreen-pHbFPS1 and effector strain pGreenII62Sk-HblMADS24 were mixed and injected into tobacco leaves for Dual-LUC assays. The HbFPS1 promoter drove luciferase expression weakly alone, while the HblMADS24 expression induced an obvious increase in luciferase activity (Fig. 8). The result indicated that HblMADS24 activated the HbFPS1 promoter expression. 


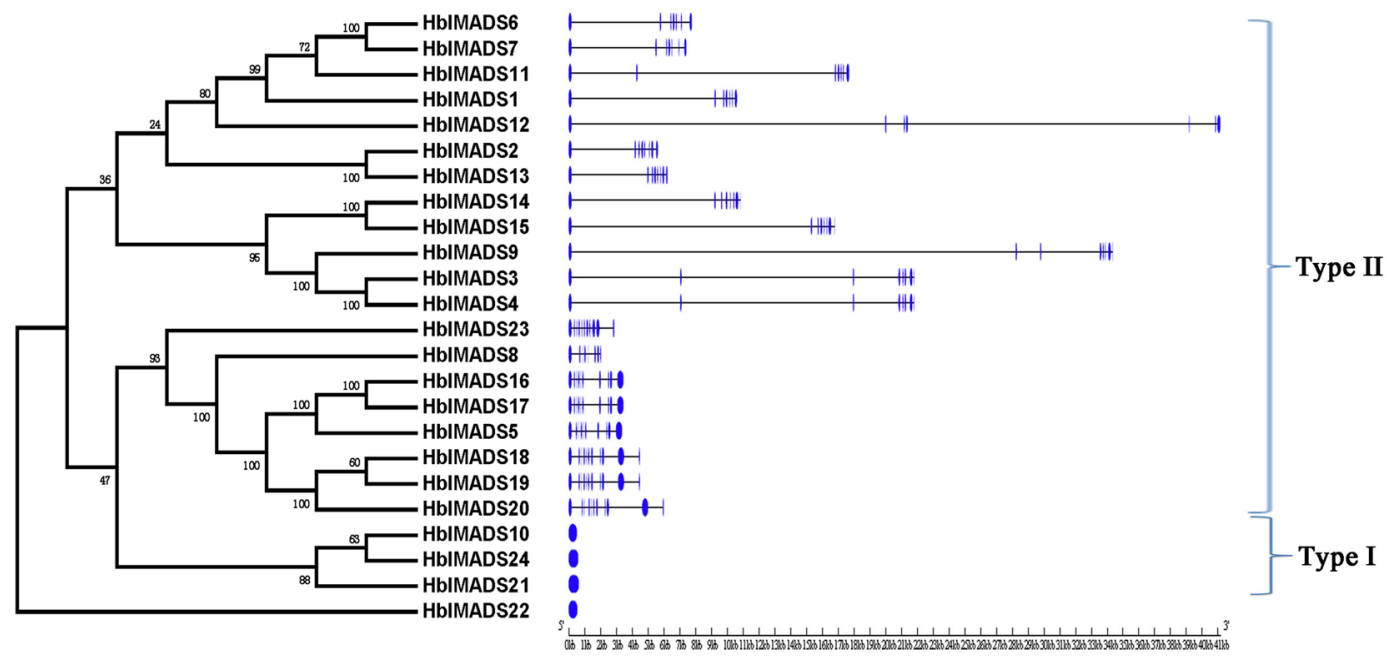

Figure 2. Phylogenetic relationships and Exon-intron structure of the MADS-box genes from H. brasiliensis. The NJ evolutionary tree was constructed with 1000 bootstrap replicates based on the alignment of full-length amino acid sequences of the MADS-box genes (left side). Meanwhile, the Exon-intron structures of the MADSbox genes are described in the right portion. Exons and introns are represented by blue and black lines. The lengths of the exons and introns of each MADS-box gene are shown proportionally.
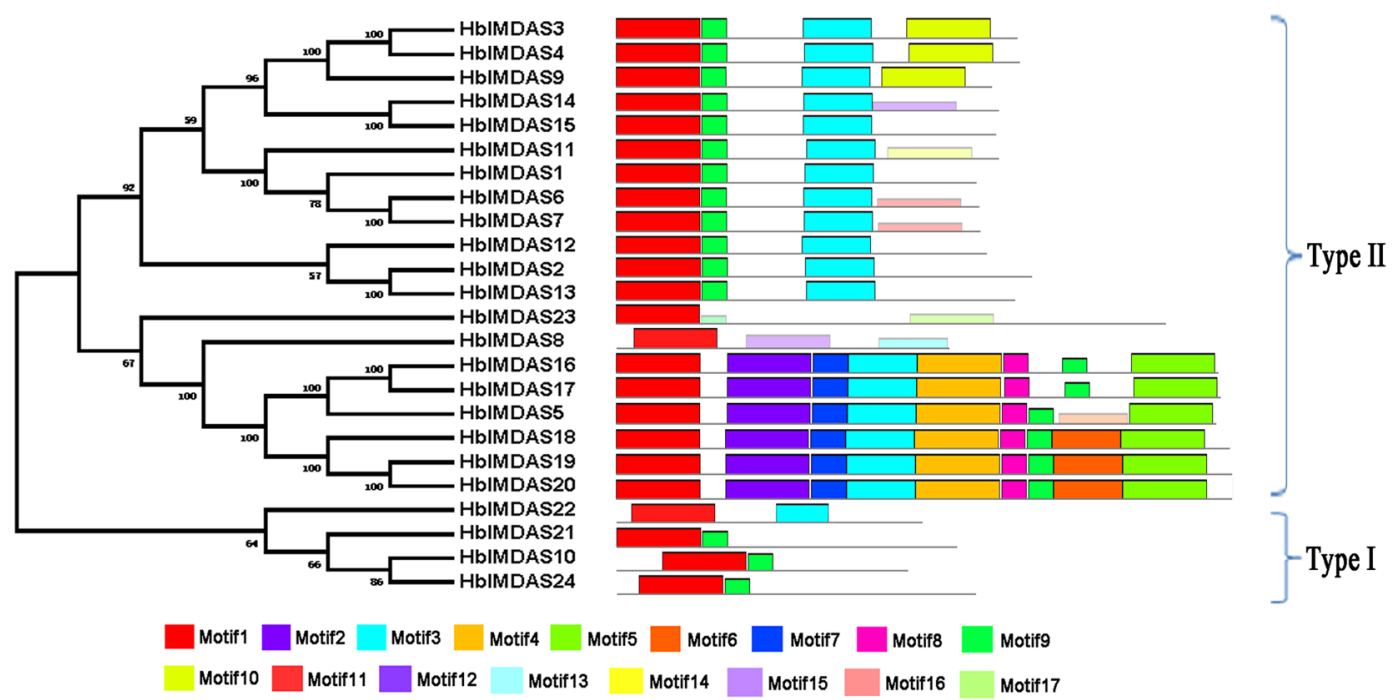

Figure 3. Phylogenetic relationships and conserved motifs of the MADS proteins from $H$. brasiliensis. The motif compositions were analyzed using MEME. Motif 1 represented MADS domain, Motif 3 represented K domain.

\section{Discussion}

The MADS-box genes have been identified and characterized in some plants species, such as Arabidopsis thaliana $^{3}$, Raphanus sativus ${ }^{9}$, Oryza sativa ${ }^{49}$, Populus trichocarpa ${ }^{50}$, Zea mays $^{51}$, Brassica rapa ${ }^{52}$, Vitis vinifera ${ }^{53}$, Malus domestica ${ }^{54}$, Prunus mume ${ }^{55}$. H. brasiliensis is a well-known rubber-producing plant used to produce commercially valuable natural rubber ${ }^{56}$. However, the characterization and systematically analysis of the MADS-box genes family has not been performed in $H$. brasiliensis.

In this study, we comprehensive analyzed 24 MADS-box family genes from the rubber tree latex transcriptome, including phylogenetic analysis, gene structures, conserved motifs distribution, expression patterns in different tissues and in response to hormone treatments, gene functional analysis. 24 MADS-box genes were classified into 8 subgroups, which provided a firm basis for better realizing the function of MADS-box genes. In addition, the function of the protein will probably change because of the different exon/intron patterns ${ }^{57}$. Twenty type II MADS-box genes had 7 to 11 exons, while four type I MADS-box genes had only one exon, which could suggest that the type II MADS-box genes contained more variable and complex function. The similar results had also been observed in Arabidopsis thaliana ${ }^{3}$, Chinese cabbage ${ }^{52}$, apple $^{54}$, Prunus mume $^{55}$, soybean ${ }^{58}$. 


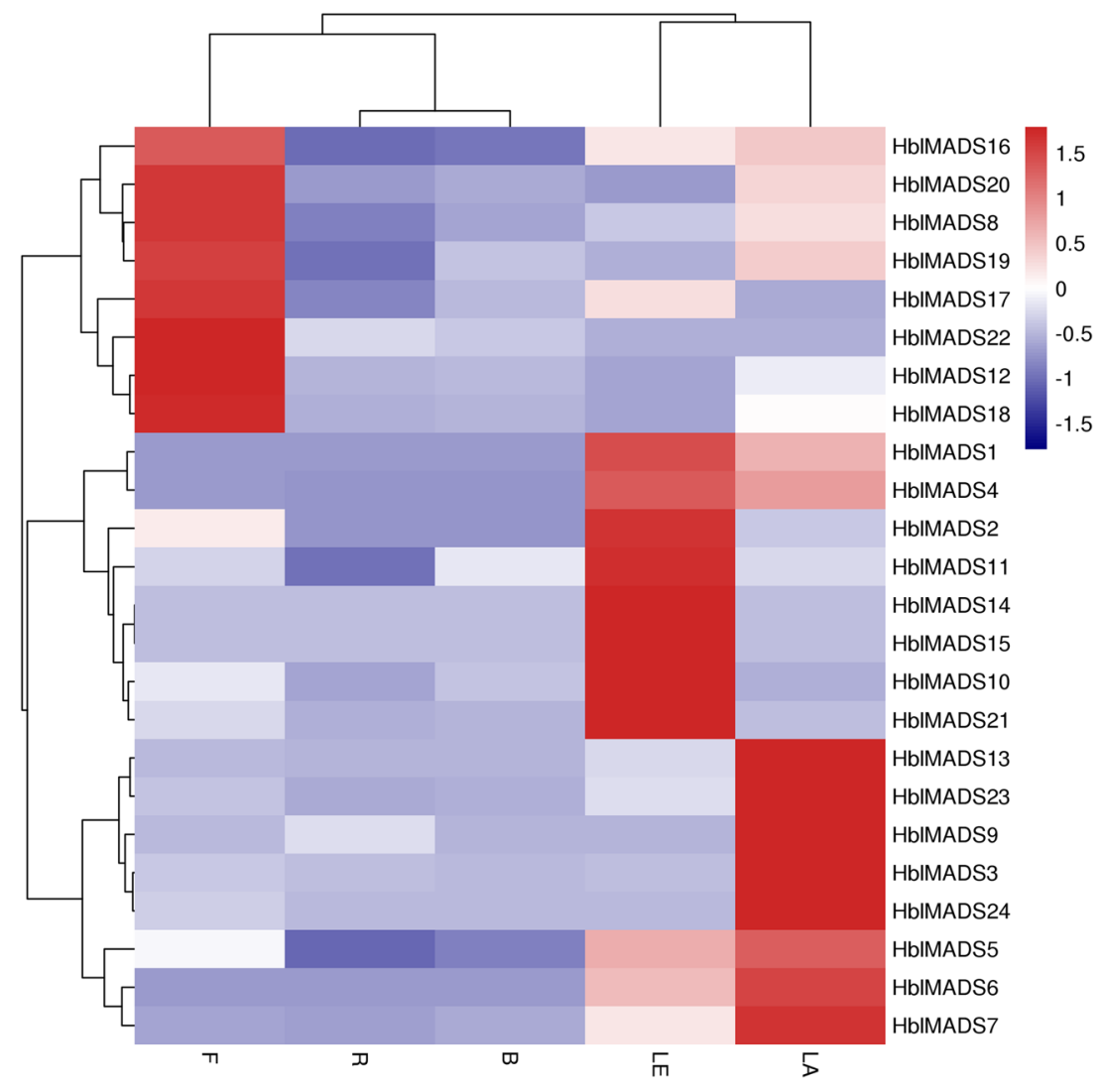

Figure 4. Heat map showing the expression levels of the MADS-box genes in five different tissues of $H$. brasiliensis. The heat map was derived from $\log _{2}$-based values of three replicates of qRT-PCR data using the online tool (http://www.omicshare. com/tools/Home/Soft/heatmap). The scale represents the relative signal intensity values. Sources of the samples are as follow: R, root; B, bark; LE, leaf; F, flower; LA, latex. The gene expression levels with different color scales are displayed at the top left corner. The groups and subgroups of MADS-box genes are displayed in the right portion.

The expression patterns of all the 24 MADS-box genes were detected in five different tissues (roots, barks, leaves, flowers, latex) by qRT-PCR. A heat map showed that most MIKC* genes were highly expressed in flowers, which were consistent with previous studies in Arabidopsis thaliana and Oryza sativa ${ }^{14,59}$. However, most $\mathrm{MIKC}^{\mathrm{C}}$ genes were highly expressed in leaf and latex. Type I displayed similar or distinct expression profiles. For instance, HblMADS24 was more expressed in latex, whereas HblMADS22 was uniquely expressed in flower, HblMADS10 and HblMADS21 was more expressed in leaf, which demonstrated that these genes may have multiple functions.

Phytohormone plays key role in NR biosynthesis ${ }^{60}$. Laticifers differentiation is specifically induced by JA ${ }^{61}$. Moreover, JA up-regulated NR biosynthesis-related genes expression, suggesting NR biosynthesis in laticifers is regulated by JA signalling ${ }^{60,62-64}$. ET has been widely applied to stimulate rubber production ${ }^{65,66}$. ABA treated rubber trees exhibited to increases in latex yield ${ }^{67}$. ABA markedly up-regulated NR biosynthesis-related genes expression, suggesting NR biosynthesis in laticifers is also regulated by ABA signalling ${ }^{68}$. SA could also induce a transient increase latex yield ${ }^{67}$. How these hormones are connected to the NR biosynthesis pathway and how their action is integrated with other regulatory circuits is currently unknown. In our present study, ABA up-regulated the expression of HblMADS9. NR biosynthesis was probably regulated by JA signaling in laticifers ${ }^{69,70}$. MeJA regulated the expression of HblMADS3, HblMADS5, HblMADS24, which indicated HblMADS3, HblMADS5, HblMADS24 may play an important role in JA signaling pathway. But the regulatory mechanism of NR biosynthesis is not clear ${ }^{71,72}$. It will be of great interest to elucidate whether MADS-box transcription factors can regulate NR biosynthesis with JA and ABA as the regulatory signals.

Compared to type II MADS-box genes, the data about type I MADS-box genes is inadequate ${ }^{73}$. Previous studies indicated that Arabidopsis type I MADS-box genes are involved in plant development and reproduction ${ }^{73-76}$. Little information is available on type I MADS-box participating in the regulation of secondary metabolism. In our present study, HblMADS24 is a typical I MADS-box gene that bound the HbFPS1 promoter. Moreover, HblMADS24 activated the HbFPS1 promoter, suggesting HblMADS24 maybe participate in the regulation of natural rubber biosynthesis. As a result, over-expression of HbFPS1 driven by HBMADS24 would increase latex yield. Identification of the MADS-box genes highly expressed in H. brasiliensis laticifers cells would greatly help to uncover the molecular regulation basis of natural rubber biosynthesis. 

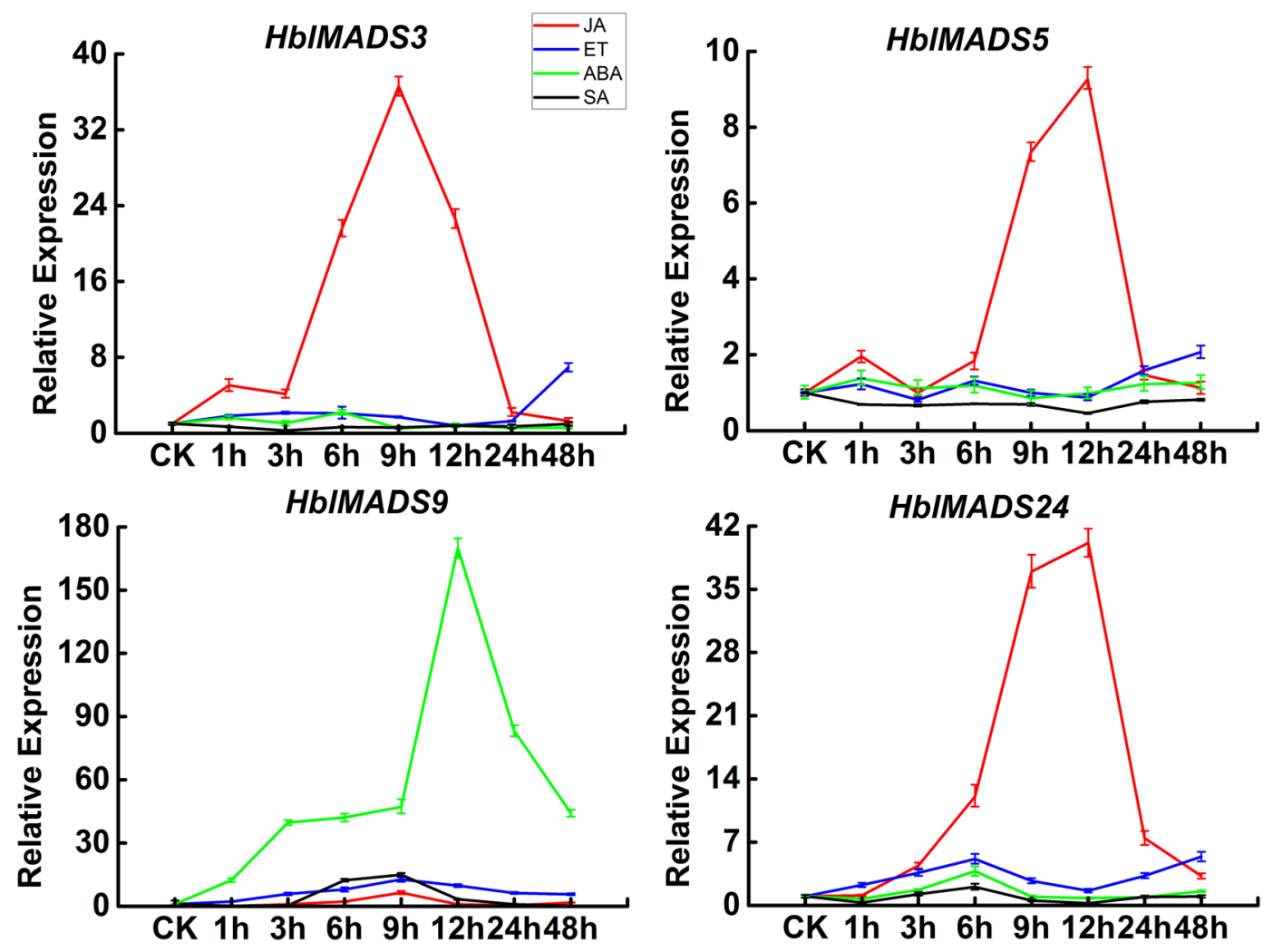

Figure 5. Expression patterns of 4 MADS-box genes responding to phytohormone treatment. RNA extracted from the latex of rubber trees treated with MeJA, ET, ABA, and SA for 0, 1, 3, 6, 9, 12, 24h and $48 \mathrm{~h}$ were subjected to RT-RCR assay. Three independent replicates were calculated to the mean at each time. The standard deviation was indicated with the vertical bars.
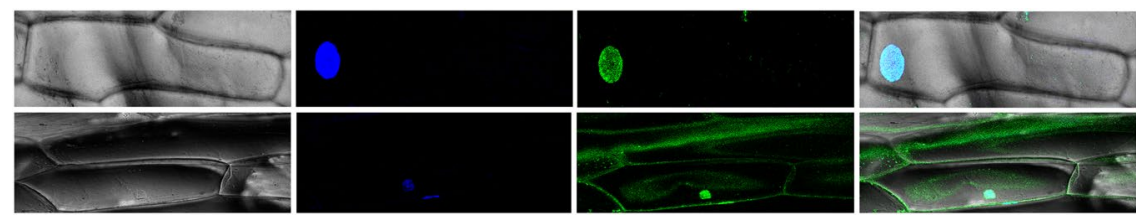

HblMADS24::GFP

Figure 6. Nuclear localization of HblMADS24. The corresponding bright-field image, DAPI image, fluorescence image, and merged image of HblMADS24-GFP were shown on the upper panel. The corresponding bright-field image, DAPI image, fluorescence image, and merged image of GFP as control were shown on the lower panel.

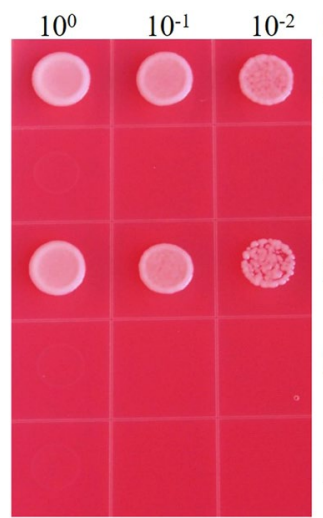

\author{
Bait/prey \\ pHis-pHbFPS1/pGAD-HblMADS24 \\ pHis-pHbFPS1/pGADT7 \\ p53His2/PGAD-53 \\ pHis 2.1/pGAD-53
}

pHis2.1/pGAD-HblMADS24

Figure 7. Activation of HbFPS1 promoter by HblMADS24 in yeast. The yeast clones harboring pGADHblMADS24 and pHis-pHbFPS1 could grow on SD/-Trp/-His/-Leu selective medium containing $70 \mathrm{mM} 3$-AT at $30^{\circ} \mathrm{C}$ for 3 days. 


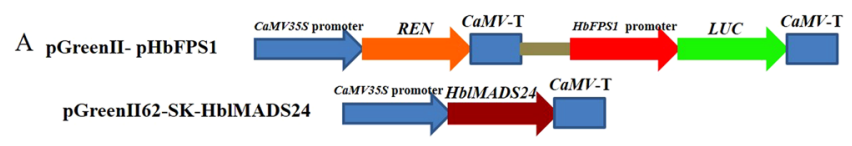

B

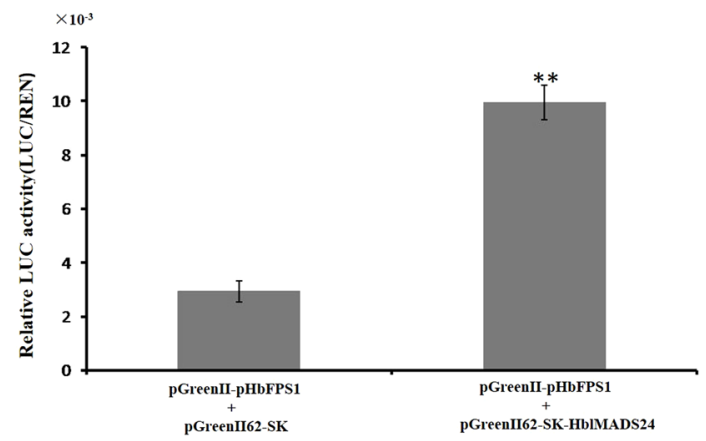

Figure 8. Transient dual-LUC was determined in tobacco leaves. (A) The expression vectors used in the transient dual-LUC assays. (B) HblMADS24 can activate the HbFPS1 promoter by transient dual-LUC analysis in tobacco leaves. The values of LUC/REN represent the level of HblMADS24 activation of the HbFPS1 promoter. The standard deviation was indicated with the error bars. Statistical significance was determined by ANOVA $(* * \mathrm{P}<0.01)$.

\section{Materials and Methods \\ Plant materials. H. brasiliensis (Wenchang11) were cultivated in the Hainan Agricultural Reclamation in Wenchang, Hainan. The shoots of two-year-old rubber trees were treated with $200 \mu \mathrm{m} \mathrm{ABA}, 200 \mu \mathrm{m} \mathrm{SA}, 0.07 \%$ JA and $0.5 \%$ ET according to the method previously ${ }^{61}$. For each hormonal treatment, the latex samples were col- lected and mixed from one group of ten-trees when treated at 1, 3, 6, 9, 12, 24 and $48 \mathrm{~h}^{77}$. One group of ten-trees without any treatment was as control. All the samples were quickly stored in the RNA extraction buffer. Four other tissues of rubber tree: roots, barks, leaves, and flowers were sampled for RNA extraction.}

Identification of the MADS-box genes in the laticifer cells. The local H. brasiliensis genome database had been established using the rubber tree genome data ${ }^{72,78}$ and NCBI-Blast-2.2.28+-win32 software in our previously study ${ }^{79}$. A total of 36 MADS-box unigenes were obtained in the rubber tree latex transcriptome database $^{47}$. The MADS-box unigenes were used as queries in searching for MADS-box genes loci in the local genome of $H$. brasiliensis using the BLASTx. Finally, MADS-box genes were obtained in the local $H$. brasiliensis genome database according to the method previously ${ }^{80}$. The molecular weight and $\mathrm{pI}$ of each HblMADS protein were predicted using ExPASy database (http://web.expasy.org/compute_pi/) ${ }^{81}$.

Phylogenetic analysis. The MADS-box transcription factor protein sequences from Arabidopsis and $O$. sativa were downloaded from the phytozome database (https://phytozome.jgi.doe.gov/pz/portal.html ${ }^{82}$. The phylogenetic tree was constructed among MADS-box proteins from $H$. brasiliensis and known MADS proteins from Arabidopsis and O. sativa according to the method previously ${ }^{80}$.

Gene structure analysis and identification of conserved motif. The online software GSDS (http:// gsds.cbi.pku.edu.cn $/)^{79}$ was utilized to reveal the exon-intron structure and coding domain sequences (CDS) of MADS-box genes from $H$. brasiliensis. The MEME (http://meme-suite.org/tools/meme) ${ }^{80}$ was employed to analyze the conserved domains of HblMADS proteins.

Expression analysis of the MADS-box genes. Latex total RNA was extracted as described previously and total RNAs from roots, barks, leaves, and flowers were isolated according to Li's method ${ }^{46}$. The first-strand cDNA was synthesized in accordance with the manual of the RevertAid ${ }^{\mathrm{TM}}$ First-Strand cDNA Synthesis Kit (Fermentas, Lithuania). The quantitative real-time PCR (qRT-PCR) was performed in accordance with the manual of the SYBR Premix EX Taq Kit (TaKaRa, Japan). The HbACT7 was amplified as the standard control ${ }^{24}$. The primers of MADS-box genes from $\mathrm{H}$. brasiliensis for RT-qPCR were listed in Additional file Table S2. The qRT-PCR reaction conditions were as follows: pre-heating at $95^{\circ} \mathrm{C}$ for $3 \mathrm{~min}$, followed by 40 cycles of $95^{\circ} \mathrm{C}$ for $10 \mathrm{~s}, 58^{\circ} \mathrm{C}$ for $20 \mathrm{~s}$, and $72^{\circ} \mathrm{C}$ for $25 \mathrm{~s}$. Three individual biological reactions were replicated. The relative expression levels were analyzed using the Stratagene Mx3005P Real Time Thermal Cycler software (Agilent, America) ${ }^{79}$.

Subcellular localization of HbIMADS24. The CDS of HblMADS24 was fused in the pCAMBIA1302 vector to generate pHblMADS24-GFP. The amplified primers were listed in Additional file Table S2. The pHblMADS24-GFP or pCAMBIA1302 was individual introduced into onion epidermal cells by Agrobacterium-mediated method. The transformed onion epiderm was placed on Murashige Skoog solid medium in darkness at $26^{\circ} \mathrm{C}$. After culturing for $5 \mathrm{~h}$, onion epiderm was observed under a confocal microscope (Zeiss LSM510, Germany).

Transcriptional activation. The HbFPS1 promoter with 975 bp nucleotides that was cloned into pHiS2.1 vector (Clontech), generating bait vector pHis-pHbFPS1. The amplified primers based on described previously $^{83}$. The CDS of HblMADS24 was fused into pGAD7 vector to generate prey vector pGAD-HblMADS24. The 
amplified primers were listed in Additional file Table S2. The transcriptional activity of HblMADS24 was detected by transforming pHis-pHbFPS1 and pGAD-HblMADS24 into yeast strain Y187 (Clontech). The introduced yeast were cultured on SD medium lacking tryptophan histidine, and leucine (SD/-Trp/-His/-Leu) adding 70 mM 3-AT at $30^{\circ} \mathrm{C}$ for $3 \mathrm{~d}$.

Agrobacterium-mediated transient expression assays. The HbFPS1 promoter and the pGreenII 0800 vector were fused to generate pGreen-pHbFPS1. The ORF of HblMADS24 was inserted the pGreenII62SK vector to form pGreenII62Sk- HblMADS24. All constructed plasmids were transformed into A. tumefaciens strain GV3103. The strain harbouring pGreen-pHbFPS1 mixed with the strain harbouring pGreenII62SK-HblMADS24 at a volume ratio of 1:6. The tobacco leaves were infiltrated with mixed A.tumefaciens. After 3 days, total proteins were extracted from the injected area of tobacco leaves. The Dual-LUC assay was conducted according to Hellens' method $^{84}$. The activity of the luciferase and REN-Luc were measured in accordance with the manual of the Dual-Luciferase Reporter Assay System (Promega). The binding activity of HblMADS24 to the HbFPS1 promoter was measured by LUC/REN. Three biological repeats were measured.

\section{References}

1. Gramzow, L. \& Theissen, G. A hitchhiker's guide to the MADS world of plants. Genome. Biol. 11, 214 (2010).

2. Smaczniak, C. et al. Characterization of MADS-domain transcription factor complexes in Arabidopsis flower development. Proc. Natl. Acad. Sci. USA 109, 1560-1565 (2012).

3. Hu, J. Y. et al. miR824-regulated AGAMOUS-LIKE16 contributes to flowering time repression in Arabidopsis. Plant Cell 26, 2024-2037 (2014).

4. Guo, X. H. et al. The MADS-box gene SlMBP11 regulates plant architecture and affects reproductive development in tomato plants. Plant Sci. 258, 90-101 (2017).

5. Schwarz-Sommer, Z. et al. Genetic control of flower development by homeotic genes in Antirrhinum majus. Science 250, 931-936 (1990).

6. Michaels, C. S. D. \& Amasino, R. M. FLOWERING LOCUS C encodes a novel MADS domain protein that acts as a repressor of flowering. Plant Cell 11, 949-956 (1999).

7. Cheng, Z. C. et al. Analysis of MADS-Box gene family reveals conservation in floral organ ABCDE model of Moso Bamboo (Phyllostachys edulis). Front. Plant. Sci. 8, 656 (2017).

8. Liu, C. et al. Specification of Arabidopsis floral meristem identity by repression of flowering time genes. Development 134, 1901-1910 (2007).

9. Li, C. et al. Genome-wide characterization of the MADS-Box gene family in Radish (Raphanus sativus L.) and assessment of its roles in flowering and floral organogenesis. Front. Plant. Sci. 7, 1390 (2016).

10. Ren, Z. Y. et al. Genome-wide identification of the MIKC-Type MADS-Box gene family in Gossypium hirsutum L. unravels their roles in flowering. Front. Plant. Sci. 8, 384 (2017).

11. Nesi, N. et al. The TRANSPARENT TESTA16 locus encodes the ARABIDOPSIS BSISTER MADS domain protein and is required for proper development and pigmentation of the seed coat. Plant Cell 14, 2463-79 (2002).

12. Liljegren, S. J. et al. SHATTERPROOF MADS-box genes control seed dispersal in Arabidopsis. Nature 404, 766-770 (2000).

13. Alvarez-Buylla, E. R. et al. An ancestral MADS-box gene duplication occurred before the divergence of plants and animals. Proc. Natl. Acad. Sci. USA 97, 5328-5333 (2000).

14. Liu, Y. et al. Functional conservation of MIKC*-Type MADS box genes in Arabidopsis and rice pollen maturation. Plant Cell 25, 1288-1303 (2013).

15. Henschel, K. et al. Two ancient classes of MIKC-type MADS-box genes are present in the moss Physcomitrella patens. Mol. Biol. Evol. 19, 801-814 (2002).

16. Kwantes, M., Liebsch, D. \& Verelst, W. How MIKC* MADS-box genes originated and evidence for their conserved function throughout the evolution of vascular plant gametophytes. Mol. Biol. Evol. 29, 293-302 (2012).

17. Becker, A. \& Theibn, G. The major clades of MADS-box genes and their role in the development and evolution of flowering plants. Mol. Phylogenet. Evol. 29, 464-489 (2003).

18. Norman, C. et al. Isolation and properties of cDNA clones encoding SRF, a transcription factor that binds to the c-fos serum response element. Cell 55, 989-1003 (1988).

19. Pellegrini, L., Tan, S. \& Richmond, T. J. Structure of serum response factor core bound to DNA. Nature 376, 490-498 (1995).

20. Shore, P. \& Sharrocks, A. D. The MADS-box family of transcription factors. Eur. J. Biochem. 229, 1-1 (1995).

21. Sasaki, K. et al. Functional divergence with in class BMADS-box genes TfGLO and TfDEF in Torenia fournieri Lind. Mol. Genet. Genomics 284, 399-414 (2010).

22. Cho, S. et al. Analysis of the C-terminal region of Arabidopsis thaliana APETALA1 as a transcription activation domain. Plant Mol. Biol. 40, 419-429 (1999).

23. De Bodt., S. et al. And then there were many: MADS goes genomic. Trends Plant Sci. 8, 475-483 (2003).

24. Smaczniak, C. et al. Developmental and evolutionary diversity of plant MADS-domain factors: insights from recent studies. Development 139, 3081-3098 (2012).

25. Acri-Nunes-Miranda, R. \& Mondragón-Palomino, M. Expression of paralogous SEP-, FUL-, AG- and STK-like MADS-box genes in wild-type and peloric Phalaenopsis flowers. Front. Plant Sci. 5, 76 (2014).

26. Ferrándiz, C., Liljegren, S. J. \& Yanofsky, M. F. Negative regulation of the SHATTERPROOF genes by FRUITFULL during Arabidopsis fruit development. Science 289, 436-438 (2000).

27. Pinyopich, A. et al. Assessing the redundancy of MADS-box genes during carpel and ovule development. Nature 424, 85-88 (2003).

28. Dreni, L. et al. The D-lineage MADS-box gene OsMADS13 controls ovule identity in rice. Plant J. 52, 690-699 (2007).

29. Li, H. et al. Rice MADS6 interacts with the floral homeotic genes SUPERWOMAN1, MADS3, MADS58, MADS13 and DROOPING LEAF in specifying floral organ identities and meristem fate. Plant Cell 23, 2536-2552 (2011).

30. Lee, H. et al. The AGAMOUS-LIKE 20 MADS domain protein integrates floral inductive pathways in. Arabidopsis. Genes Dev. 14, 2366-2376 (2000).

31. Hepworth, S. R. et al. Antagonistic regulation of flowering-time gene SOC1 by CONSTANS and FLC via separate promoter motifs. EMBO J. 21, 4327-4337 (2002).

32. Searle, I. et al. The transcription factor FLC confers a flowering response to vernalization by repressing meristem competence and systemic signaling in. Arabidopsis. Genes Dev. 20, 898-912 (2006).

33. Reeves, P. A. et al. Evolutionary conservation of the FLOWERING LOCUS Cmediated vernalization response: evidence from the sugar beet (Beta vulgaris). Genetics 176, 295-307 (2007).

34. Liu, C. et al. Direct interaction of AGL24 and SOC1 integrates flowering signals in Arabidopsis. Development 135, 1481-1491 (2008). 
35. Michaels, S. D. et al. AGL24 acts as a promoter of flowering in Arabidopsis and is positively regulated by vernalization. Plant J. 33, 867-874 (2003).

36. Lee, J. H. et al. Role of SVP in the control of flowering time by ambient temperature in. Arabidopsis. Genes Dev. 21, 397-402 (2007).

37. Kush, A. Isoprenoid biosynthesis: the Hevea factory. Plant Physiol. Biochem. 32, 761-767 (1994).

38. Archer, B. L. \& Audley, B. G. New aspects of rubber biosynthesis. Bot. J. Linn. Soc. 94, 181-196 (1987).

39. Chow, K. S. et al. Insights into rubber biosynthesis from transcriptome analysis of Hevea brasiliensis latex. J. Exp. Bot. 58, 2429-2440 (2007).

40. Chow, K. S. et al. Metabolic routes affecting rubber biosynthesis in Heveabrasiliensis latex. J. Exp. Bot. 63, 1863-1871 (2012).

41. Sando, T. et al. Cloning and characterization of mevalonate pathway genes in a natural rubber producing plant, Hevea brasiliensis. Biosci. Biotechnol. Biochem. 72, 2049-2060 (2008).

42. Tangpakdee, T. \& Tanaka, Y. Long-chain polyprenols and rubber in young leaves of Hevea brasiliensis. Phytochemistry 48, 447-450 (1998).

43. Guo, D., Li, H. L. \& Peng, S. Q. Structure conservation and differential expression of farnesyl diphosphate synthase genes in Euphorbiaceous plants. Int. J. Mol. Sci. 16, 22402-22414 (2015).

44. Wang, Y. et al. Characterization of HbWRKY1, a WRKY transcription factor from Hevea brasiliensis that negatively regulates HbSRPP. Plant Physiol. Biochem. 71, 283-289 (2013).

45. Li, H. L. et al. Three MADS-box genes of Hevea brasiliensis expressed during somatic embryogenesis and in the laticifer cells. Mol. Biol. Rep. 38, 4045-4052 (2011).

46. Li, H. L. et al. HbMADS4, a MADS-box transcription factor from Hevea brasiliensis, negatively regulates HbSRPP. Front. Plant Sci. 7, 1709 (2016a).

47. Li, H. L. et al. Comparative transcriptome analysis of latex reveals molecular mechanisms underlying higher rubber yield in Hevea brasiliensis self-rooting juvenile clones. Front. Plant Sci. 7, 1204 (2016b).

48. Lyons, E. et al. Finding and comparing syntenic regions among Arabidopsis and the outgroups papaya, poplar, and grape: CoGe with rosids. Plant Physiol. 148, 1772-1781 (2008).

49. Arora, R. et al. MADS-box gene family in rice: genome-wide identification, organization and expression profiling during reproductive development and stress. BMC Genomics 8, 242 (2007).

50. Leseberg, C. H. et al. Genome-wide analysis of the MADS-box gene family in Populus trichocarpa. Gene 378, 84-94 (2006).

51. Zhao, Y. et al. Whole-genome survey and characterization of MADS-box gene family in maize and sorghum. Plant Cell Tissue Organ. Cult. 105, 159-173 (2010)

52. Duan, W. et al. Genome-wide analysis of the MADS-box gene family in Brassica rapa (Chinese cabbage). Mol. Genet. Genomics 290, 239-255 (2015)

53. Diaz-Riquelme, J. et al. Genome-wide analysis of MIKCC-type MADS box genes in grapevine. Plant Physiol. 149, 354-369 (2009).

54. Velasco, R. et al. The genome of the domesticated apple (Malus $x$ domestica Borkh.). Nat. Genet. 42, 833-839 (2010).

55. Xu, Z. D. et al. Genome-wide identification, characterisation and expression analysis of the MADS- box gene family in Prunus mume. Mol. Genet. Genomics 289, 903-920 (2014).

56. Van Beilen., J. B. \& Poirier, Y. Establishment of new crops for the production of natural rubber. Trends Biotechnol. 2, 522-529 (2007).

57. Tian, Y. et al. Genome-wide identification and analysis of the MADS-box gene family in apple. Gene 555, 277-290 (2015).

58. Fan, C. M. et al. Genome-wide expression analysis of soybean MADS genes showing potential function in the seed development. PloS one 8, e62288 (2013).

59. Verelst, W., Saedler, H. \& Münster, T. MIKC* MADS-protein complexes bind motifs enriched in the proximal region of late pollenspecific Arabidopsis promoters. Plant Physiol. 14, 447-460 (2007).

60. Deng, X. et al. Jasmonate signalling in the regulation of rubber biosynthesis in laticifer cells of rubber tree, Hevea brasiliensis. J Exp. Bot. 69, 3559-3571 (2018).

61. Hao, B. Z. \& Wu, J. L. Laticifer differentiation in Hevea brasiliensis: induction by exogenous jasmonic acid and linolenic acid. Ann. Bot. 85, 37-43 (2000)

62. Peng et al. Cloning and molecular characterization of HbCOI1 from Hevea brasiliensis. Biosci. Biotechnol. Biochem. 73, 665-670 (2009).

63. Tian, W. W., Huang, W. F. \& Zhao, Y. Cloning and characterization of HbJAZ1 from the laticifer cells in rubber tree (Hevea brasiliensis Muell. Arg.). Trees 24, 771-779 (2010).

64. Pirrello, J. et al. Transcriptional and post-transcriptional regulation of the jasmonate signaling pathway in response to abiotic and harvesting stress in Hevea brasiliensis. BMC Plant Biol. 14, 341 (2014).

65. Zhu, J. \& Zhang, Z. Ethylene stimulation of latex production in Hevea brasiliensis. Plant Signal Behav. 4, 1072-1074 (2009).

66. Tungngoen, K. et al. Involvement of HbPIP2;1 and HbTIP1;1 aquaporins in ethylene stimulation of latex yield through regulation of water exchanges between inner liber and latex cells in Hevea brasiliensis. Plant Physiol. 151, 843-856 (2009).

67. Tungngoen, K. et al. Hormonal treatment of the bark of rubber trees (Hevea brasiliensis) increases latex yield through latex dilution in relation with the differential expression of two aquaporin genes. J Plant Physiol. 168, 253-262 (2011).

68. Guo, D. et al. Identification and characterization of the abscisic acid (ABA) receptor gene family and its expression in response to hormones in the rubber tree. Sci. Rep. 7, 45157 (2017).

69. Zeng, R. Z. et al. Vacuolar type inorganic pyrophosphatase located on the rubber particle in the latex is an essential enzyme in regulation of the rubber biosynthesis in Hevea brasiliensis. Plant Sci. 176, 602-607 (2009).

70. Pirrello, J. et al. Transcriptional and post-transcriptional regulation of the jasmonate signalling pathway in response to abiotic and harvesting stress in Hevea brasiliensis. BMC Plant Biol. 14, 341 (2014).

71. Yamashita, S. et al. Identification and reconstitution of the rubber biosynthetic machinery on rubber particles from Hevea brasiliensis. eLife 5, e19022 (2016).

72. Tang, C. R. et al. The rubber tree genome reveals new insights into rubber production and species adaptation. Nat. Plants 2, 16073 (2016).

73. Bouyer, D. et al. Polycomb repressive complex 2 controls the embryo-to-seedling phase transition. PLoS Genet. 7, e1002014 (2011).

74. Kang, I. H. et al. The AGL62 MADS domain protein regulates cellularization during endosperm development in Arabidopsis. Plant Cell 20, 635-647 (2008).

75. Bemer, M. et al. The MADS domain protein DIANA acts together with AGAMOUS-LIKE80 to specify the central cell in Arabidopsis ovules. Plant Cell 20, 2088-2101 (2008).

76. Portereiko, M. F. et al. AGL80 is required for central cell and endosperm development in Arabidopsis. Plant Cell 18, 1862-1872 (2006).

77. Tang, C. R. et al. A convenient and efficient protocol for isolating high-quality RNA from latex of Hevea brasiliensis (para rubber tree). J. Biochem. Biophys. Methods 70, 749-754 (2007).

78. Rahman, A. Y. et al. Draft genome sequence of the rubber tree (Hevea brasiliensis). BMC Genomics 14, 75 (2013).

79. Wang, Y. et al. Transcriptome-wide identification and characterization of MYB transcription factor genes in the laticifer cells of Hevea brasiliensis. Front. Plant Sci. 8, 1974 (2017).

80. Zhang, L. et al. Genome-wide identification, characterization of the MADS-box gene family in Chinese jujube and their involvement in flower development. Sci. Rep. 7, 1025 (2017).

81. Artimo, P. et al. ExPASy: SIB bioinformatics resource portal. Nucleic Acids Res. 40, W597-603 (2012). 
82. Goodstein, D. M. et al. Phytozome: a comparative platform for green plant genomics. Nucleic Acids Res. 40, D1178-86 (2012).

83. Guo, D., Li, H. L. \& Peng, S. Q. Cloning and function analysis of 5 ' regulatory region for HbFPS from Hevea brasiliensis. Trop. Agri. Engineer. 34, 33-36 (2010).

84. Hellens, R. G. et al. Transient expression vectors for functional genomics, quantification of promoter activity and RNA silencing in plants. Plant Methods 1, 13 (2005)

\section{Acknowledgements}

This work was supported by grants of National Key R\&D Program of China (No. 2018YFD1000502) and National Natural Science Foundation of China (No. 31670611).

\section{Author Contributions}

S.Q.P. conceived the study. Y.W., D.F.Z., H.L.L., D.G. and J.H.Z. performed the experiments and carried out the analysis. Y.W. and S.Q.P. designed the experiments and wrote the manuscript. All authors read and approved the final manuscript.

\section{Additional Information}

Supplementary information accompanies this paper at https://doi.org/10.1038/s41598-019-48958-9.

Competing Interests: The authors declare no competing interests.

Publisher's note: Springer Nature remains neutral with regard to jurisdictional claims in published maps and institutional affiliations.

(1) Open Access This article is licensed under a Creative Commons Attribution 4.0 International License, which permits use, sharing, adaptation, distribution and reproduction in any medium or format, as long as you give appropriate credit to the original author(s) and the source, provide a link to the Creative Commons license, and indicate if changes were made. The images or other third party material in this article are included in the article's Creative Commons license, unless indicated otherwise in a credit line to the material. If material is not included in the article's Creative Commons license and your intended use is not permitted by statutory regulation or exceeds the permitted use, you will need to obtain permission directly from the copyright holder. To view a copy of this license, visit http://creativecommons.org/licenses/by/4.0/.

(c) The Author(s) 2019 